ISSUES IN MEDICINE

\title{
Preventing hepatitis $B$ and hepatocellular carcinoma in South Africa: The case for a birth-dose vaccine
}

\author{
C W N Spearman, M W Sonderup \\ Wendy Spearman heads and Mark Sonderup is the senior consultant in the Division of Hepatology, Department of Medicine, Faculty of \\ Health Sciences, University of Cape Town, South Africa.
}

Corresponding authors: CW N Spearman (wendy.spearman@uct.ac.za), MW Sonderup (msonderup@samedical.co.za)

\begin{abstract}
Hepatitis B is a global public health issue, with some 2 billion people having current or past infection. In Africa, 65 million are chronically infected, an estimated 2.5 million of them in South Africa (SA). Hepatitis B and the associated complications of cirrhosis and hepatocellular carcinoma are entirely vaccine preventable. SA was one of the first ten countries in Africa to introduce universal hepatitis B vaccination in April 1995, but has no birth dose or catch-up programme. Although universal infant vaccination in SA has been successful in increasing population immunity to hepatitis $\mathrm{B}$, improvements in terms of implementing protocols to screen all pregnant mothers for hepatitis $\mathrm{B}$ surface antigen $(\mathrm{HBs} \mathrm{Ag})$ and ensuring full hepatitis B coverage, especially in rural areas, is required. The World Health Organization has recommended a birth dose of hepatitis B vaccine in addition to the existing hepatitis B vaccine schedule in order to further decrease the risk of perinatal transmission. We recommend that SA implement a birth-dose vaccine into the existing schedule to attenuate the risk of perinatal transmission, prevent breakthrough infections and decrease HBsAg carriage in babies born to HIV-positive mothers.
\end{abstract}

S Afr Med J 2014;104(9):610-612. DOI:10.7196/SAMJ.8607

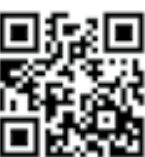

Globally some 250 - 400 million people are chronically infected with hepatitis B, with sub-Saharan Africa (sSA) and South-East Asia being disproportionately affected. Compared with the 1.5 million deaths annually due to HIV/AIDS, which are declining, hepatitis B mortality is on the rise with $500000-1.2$ million deaths annually. This relates in part to hepatocellular carcinoma (HCC), the fifth most common malignancy and the third leading cause of cancer-related death worldwide, despite the fact that hepatitis B is an entirely vaccine-preventable disease. ${ }^{[1]}$

\section{Burden of disease}

The prevalence of hepatitis B varies geographically, with $45 \%$ of the global population living in endemic areas $(\geq 8 \%$ prevalence of hepatitis B surface antigen (HBsAg)), notably the Asia-Pacific and sSA region. ${ }^{[1,2]}$ An estimated 65 million people in Africa are chronically infected, 2.5 million of them in South Africa (SA). ${ }^{[3,4]}$ In endemic countries, the majority of infections occur either perinatally or horizontally during early childhood and mostly before the age of 5 years, although in hepatitis B e-antigen ( $\mathrm{HBeAg}$ )-positive mothers, perinatal transmission is considerably higher. The risk of chronicity depends on the age of acquisition, decreasing from $70-90 \%$ with perinatal infection to 25 - 60\% in early childhood, 6 - 10\% for ages $5-20$ years and $1-5 \%$ for adults aged $>20$ years. ${ }^{[1]}$ In SA, horizontal child-to-child transmission dominates from 6 months to 5 years of age and seroprevalence varies between genders, between ethnic groups and between urban and rural areas. ${ }^{[4-7]}$ Highlighting this, an Eastern Cape Province community-based cross-sectional HBsAg prevalence study of 2299 children aged 0 - 6 years before the introduction of hepatitis B immunisation demonstrated that $10.4 \%$ children were HBsAg-positive, increasing from $8.1 \%$ at $0-6$ months to $15.7 \%$ at $61-72$ months. ${ }^{[8]}$ Adult-acquired hepatitis B is poorly understood in SA, but probably represents a relatively small component of the chronic hepatitis B virus (HBV)-infected population.

Given that 15 - 25\% of infected patients may develop cirrhosis, liver failure or hepatocellular carcinoma, there is a need for improved surveillance of acute and chronic HBV infection in Africa to better understand the economic burden of HBV infection and its complications. In SA there is the dual burden of hepatitis B and HIV, although with current antiretroviral regimens containing effective anti-HBV drugs, those with co-infection are at least accessing therapy. The HIV programme does not automatically test for HBsAg, which is unfortunate given that surveillance data would provide epidemiological information and allow for targeted interventions such as vaccinating partners of those infected with hepatitis $B$. Equally, no antenatal testing is performed, therefore not allowing for measures to reduce the risk of mother-to-child transmission of HBV.

\section{Hepatocellular carcinoma}

HCC is a significant cause of cancer mortality in sSA, and 46000 new cases are diagnosed annually. The age-standardised incidence of HCC is as high as 41.2/100 000 persons/year, with prognosis being poor and $92 \%$ dying within 1 year of the onset of symptoms. ${ }^{[8]} \mathrm{HBV}$, a vaccine-preventable disease, accounts for $60-80 \%$ of HCC, which can develop even in the absence of cirrhosis. Furthermore, given that the HBV DNA incorporates itself into the host hepatocyte genome, even those with occult hepatitis B virus infection (i.e. HBsAgnegative with detectable serum HBV DNA), or those with previous exposure, are at risk of HCC. ${ }^{[9]}$

\section{Vaccination}

The seroprevalence of HBsAg in SA before the introduction of hepatitis B vaccination in 1995 ranged from $0.2 \%$ to $9.6 \%$, with serological evidence of past exposure to hepatitis B (HBsAg-negative, 
$\mathrm{HB}$ core IgG-positive) ranging from $5 \%$ to $76 \% .^{[3,5,6]}$ Marked differences between ethnic groups were noted. HBsAg rates were considerably higher in rural areas: $15.5 \%$ in the Eastern Cape (former rural Transkei), compared with $7.4 \%$ in urban Durban and $1.3 \%$ in Soweto. In rural areas, $\mathrm{HBeAg}$ seroprevalence was also higher in HBsAg-positive mothers: $12 \%$ in HBsAg-positive mothers from rural areas compared with $0 \%$ in $\mathrm{HBsAg}$-positive pregnant women in Soweto. A 2.6:1 male/female predominance is well documented. ${ }^{[5,7]}$

Universal infant vaccination is the most effective way to reduce the global burden of HBV, and the World Health Organization (WHO) recommended its incorporation into the Expanded Programme of Immunization (EPI) in 1991. ${ }^{[10,11]}$ This proved exemplary in Taiwan, where universal vaccination, introduced in 1984, together with a catch-up vaccination programme and improved maternal screening, resulted in a decrease in the prevalence of HBsAg positivity in children aged $<15$ years from $9.8 \%$ in 1984 to $0.7 \%$ in $1999 .{ }^{[12,13]}$ Furthermore, the prevalence of HCC in children aged 6 - 9 years decreased from 5.2 cases/million population in 1984 to $1.3 /$ million in the first vaccination cohort. ${ }^{[14,15]}$ To date, 183 countries worldwide and 45 in the WHO Africa region have incorporated hepatitis B vaccination into the EPI. It is estimated that this has prevented more than 1.3 million deaths. The global vaccination coverage with three doses is $75 \%$ (41-89\%), while birth-dose coverage occurs in 94 WHO member countries. Of concern is low birth-dose coverage in countries where there is a high risk of perinatal and early childhood transmission. At present, 38 out of 56 Global Alliance for Vaccines and Immunization (GAVI)-eligible countries are not providing a birth dose, and in Africa birth coverage stands at only $23 \%$, with full coverage with three doses, not including a birth dose, at only 67\% (50 - 79\%). In South-East Asia, the rate of birth coverage is $10 \%$ and that of full coverage $41 \% .^{[1,16]}$ The 2010 official vaccine coverage rate of $97 \%$ in SA is probably overestimated given the potentially reduced coverage in rural areas and a WHO reported coverage of $56 \%$ in $2007 . .^{[17]}$

Birth-dose coverage is important in preventing perinatal transmission. This has been demonstrated in China, where a partnership between GAVI and the Chinese government supported a free birthdose vaccine; in combination with the up-scaling of the full vaccine schedule, $<1 \%$ of Chinese children are now hepatitis B-infected. ${ }^{[18]}$ SA was one of the first 10 countries in Africa to introduce universal HBV vaccination (6-, 10- and 14-week schedule) in April 1995, but currently has no birth dose and no catch-up programme. Obvious benefit has already been achieved, with the overall seroprevalence of HBsAg declining from $12.8 \%$ to $3 \%$ in some studies. A 2 - 3-year follow-up of 186 infants vaccinated in 1995 with Hepaccine B showed seroprotection (anti-hepatitis B surface antibody (anti-HBsAb) $\geq 10 \mathrm{mIU} / \mathrm{ml}$ ) in $93 \%$ and $76.8 \%$ initially and 3 years later, respectively. No children were positive for HBsAg, HBV DNA or anti-hepatitis B core antibody (anti-HbcoreAb). ${ }^{[19]}$ In the Eastern Cape, of 1213 fully vaccinated 12 - 24-month-old infants born after 1995, none were HBsAg-positive, $0.9 \%$ (9/986) were anti-HBcoreAb-positive, $0.3 \%$ (4/1 213) were HBV DNA-positive and $84.6 \%$ (834/986) had protective anti-HBsAb levels. In contrast, 7.8\% (39/498) unvaccinated 12 - 24-month-old infants born before 1995 were HBsAg-positive, $1.9 \%(4 / 203)$ were anti-HBcoreAb-positive, and 6.5\% (30/459) had occult hepatitis B infection. ${ }^{[20]}$ Another study assessed the efficacy of universal vaccination 5 years after the implementation of the programme in 598 infants from Limpopo Province aged 8 - 72 months (mean 23.3). Of the infants, $86.8 \%(519 / 598)$ were anti-HBsAbpositive (titre $\geq 10 \mathrm{mIU} / \mathrm{ml}$ ), $0 \%$ HBsAg-positive and $0 \% \mathrm{HBV}$ DNApositive; however, $0.9 \%(5 / 582)$ of infants aged $8-11$ months were
anti-HBcoreAb-positive. ${ }^{[22]}$ Similarly, assessment of the efficacy of universal HBV vaccination in 770 healthy 18 -month-old babies from rural areas of the nine provinces 1 year after vaccination revealed that $87 \%$ were anti-HBsAb-positive (titre $\geq 10 \mathrm{mIU} / \mathrm{ml}$ ), $0.4 \% \mathrm{HBsAg}$ positive and $0.5 \%$ anti-HBcoreAb-positive, compared with historical controls of $10.1 \%$ HBsAg seroprevalence in children aged $0-6$ years. ${ }^{[23]}$

\section{Influence of HIV}

HIV/HBV co-infection increases the risk of perinatal transmission. Reduced seroprotection in under-2-year-old HIV-positive v. negative children has been demonstrated. Here $78.1 \%$ (57/73) v. $85.7 \%$ $(197 / 230)$ were anti-HBsAb-positive (titre $\geq 10 \mathrm{mIU} / \mathrm{ml}$ ) and $2.7 \%$ $(2 / 73)$ v. $0.4 \%(1 / 230)$ HBsAg-positive, with an equivalent anti$\mathrm{HBcore} \mathrm{Ab}$ positivity of $3 \%$ and $2.7 \%$, respectively. ${ }^{[24]} \mathrm{A} 2007$ antenatal study comparing $1420 \mathrm{HIV}$-positive and negative mothers noted higher anti-HBcoreAb positivity (39.2\% v. $30.1 \%)$ in HIV-infected women, while $6.2 \%$ were HBsAg-positive. ${ }^{[25]}$

HIV also reduces transfer of maternal anti-HBs. Only $21 \%$ of HIVexposed v. $54 \%$ of unexposed babies had protective levels of anti-HBs, suggesting that $79 \%$ of babies born to HIV-positive mothers have no protective anti-HBs until after the first hepatitis $B$ vaccination at 6 weeks of age. ${ }^{[3,26]}$ Breakthrough infections can occur, mainly in HIVexposed/infected babies. ${ }^{[24]}$

In 9355 pregnant women from antenatal clinics in the Western Cape Province, no difference was seen between prevalences of HBsAg in HIV-positive and negative women (3.4\% (53/1 543) v. $2.9 \%(44 / 1546))$ and $\mathrm{HBeAg}(18.9 \%(10 / 53)$ v. $17.1 \%(7 / 41))$. However, HBV DNA levels were much higher in HIV-positive women, at $9.72 \times 10^{7} \mathrm{IU} / \mathrm{ml}$ v. $1.19 \times 10^{6} \mathrm{IU} / \mathrm{ml}$ in HIV-negative women, implying that the risk of perinatal transmission would be elevated. ${ }^{[27]}$

\section{Need for a birth-dose vaccine}

Although universal infant vaccination in SA has demonstrated increased population immunity to hepatitis B, there is still significant room for improvement given the absence of screening of pregnant women for HBsAg as well as the absence of a catch-up vaccination programme such as that implemented in Taiwan. Full coverage with three doses needs to be achieved, especially in rural areas where HBsAg seroprevalence is highest. To prevent perinatal transmission, a birth dose of the vaccine preferably needs to be administered within 12 hours of delivery, and certainly within 24 hours. The hepatitis B monovalent vaccine can be administered together with oral polio and BCG. SA must consider a four-dose schedule consisting of a monovalent birth dose followed by three doses of monovalent vaccine, given together with the routine infant vaccines or as a combination vaccine at 6,10 and 14 weeks. Challenges will be faced in rural areas where there is a higher prevalence of hepatitis $\mathrm{B}$, home births occasionally occur and birth BCG coverage is lower $(70-79 \%) .{ }^{[3]}$ Studies have confirmed the thermostability of HBV vaccines outside the cold chain, assisting access to the birth dose in rural areas. ${ }^{[29]} \mathrm{A}$ four-dose schedule, essentially requiring the addition of a birth dose to the existing schedule, is slightly more costly than a three-dose schedule. However, it is easier to implement and does not immunologically compromise infants who may not access a birth dose. A four-dose approach is also recommended for improved immunogenicity if penta- or hexavalent vaccines are used in the EPI schedule.

It is unfortunate that recent a request to GAVI, co-signed by 76 global groups, to fund a birth-dose vaccine in all countries not undertaking this routinely, has not been favourably received. 


\section{Adolescent booster vaccine}

Immunity from vaccination, although good, tends to decline at a time when there is increased risk of acquisition of hepatitis B due to sexual activity or risky lifestyle behaviour such as injecting drug use. A Chinese study assessed the long-term efficacy of postnatal active-passive vaccination (HBIG) in 8733 high-school students. Among those who did not receive HBIG, there was a significant negative association between hepatitis $B$ vaccination dosage and HBsAg positivity. The adjusted odds ratios for those who received 4, 3 and 1 - 2 doses were 1.00, 1.52 (95\% confidence interval (CI) $0.91-2.53)$ and 2.85 (95\% CI $1.39-5.81$ ), respectively. Notably, onesixth of students who had received four-dose coverage had lost their immunological memory against $\mathrm{HBsAg}$ by the age of 15 years. ${ }^{[28]}$

\section{Conclusion}

A strong case exists for the implementation of a birth dose of hepatitis $B$ vaccine in addition to the current schedule, as recommended by the WHO, to decrease the risk of perinatal transmission, prevent breakthrough infections, and decrease HBsAg carriage in HIVpositive babies. An adolescent booster dose, although not routinely recommended, merits consideration, as immunological memory against HBsAg is lost in a significant number of adolescents by the age of 15 years.

1. World Health Organization Position Paper. Hepatitis B vaccines. WHO Wkly Epidemiol Rec 2009;84(40):405-420. http://www.who.int/wer/2009/wer8440.pdf?ua=1 (accessed 16 June 2014)

2. Kramvis A, Kew MC. Epidemiology of hepatitis B virus in Africa, its genotypes and clinical associations of genotypes. Hepatol Res 2007;37(Suppl 1):S9-S19. [http://dx.doi.org/10.1111/j.1872 034X.2007.00098.x]

3. Burnett RJ, Kramvis A, Dochez C, Meheus A. An update after 16 years of hepatitis B vaccination in South Africa. Vaccine 2012;30(Suppl 3):C45-C51. [http://dx.doi.org/10.1016/j.vaccine.2012.02.021]

4. Robson SC, Kirsch RE. National strategy for viral hepatitis: Recommendations and guidelines for Robson SC, Kirsch RE. National strategy for viral hepatitis:
management in South Africa. S Afr Med J 1991;80(7):347-356.

5. Kire CF. The epidemiology and prophylaxis of hepatitis B in sub-Saharan Africa: A view from tropical and subtropical Africa. Gut 1996;38(Suppl 2):S5-S12. [http://dx.doi.org/10.1136/gut.38.Suppl_2.S5] 6. Kew MC. Hepatitis B virus infection: The burden of disease in South Africa. Southern African Journal 6. Kew MC. Hepatitis B virus infection: The burden
of Epidemiology and Infection 2008;23(1):4-8.

7. Vardas E, Mathai M, Blaauw D, McAnerney J, Coppin A, Sim J. Preimmunization epidemiology of hepatitis B virus infection in South African children. J Med Virol 1999;58(2):111-115. [http://dx.doi org/10.1002/(SICI) 1096-9071(199906)58:2<111::AID-JMV2 >3.0.CO;2-B]

8. Kew MC. Epidemiology of hepatocellular carcinoma in sub-Saharan Africa. Ann Hepatol 2013;12(2):173-182.

9. Kew MC, Welschinger R, Viana R. Occult hepatitis B virus infection in Southern African blacks with hepatocellular carcinoma. J Gastroenterol Hepatol 2008;23(9):1426-1430. [http://dx.doi.org/10.1111/ j.1440-1746.2008.05481.x]
10. Beutels P. Economic evaluations of hepatitis B immunization: A global review of recent studies (19942000). Health Econ 2001;10(8):751-774. [http://dx.doi.org/10.1002/hec.625]

11. Lavanchy D. Hepatitis B virus epidemiology, disease burden, treatment, and current and emerging pavanchy D. Hepatitis B virus epidemiology, disease burden, treatment, and current and emerging
prevention and control measures. J Viral Hepat 2004;11(2):97-107. [http://dx.doi.org/10.1046/.13652893.2003.00487.x]

12. Chen DS, Hsu NH, Sung JL, et al. A mass vaccination program in Taiwan against hepatitis B virus infection in infants of hepatitis B surface antigen-carrier mothers. JAMA 1987;257(19):2597-2603.
interis [http://dx.doi.org/10.1001/jama.1987.03390190075023]

13. Hsu HM, Chen DS, Chuang CH, et al. Efficacy of a mass hepatitis B vaccination program in Taiwan: Studies on 3464 infants of hepatitis B surface antigen-carrier mothers. JAMA 1988;260(15):2231-2235. [http://dx.doi.org/10.1001/jama.260.15.2231]

14. Chen HL, Chang MH, Ni YH, et al. Seroepidemiology of hepatitis B virus infection in children: Ten years of mass vaccination in Taiwan. JAMA 1996;276(11):906-908. [http://dx.doi.org/10.1001/ jama.276.11.906]

15. Ni YH, Chang MH, Huang LM, et al. Hepatitis B virus infection in children and adolescents in a hyperendemic area: 15 years after mass hepatitis B vaccination. Ann Intern Med 2001;135(9):796-800. [http://dx.doi.org/10.7326/0003-4819-135-9-200111060-00009]

16. World Health Organization. Immunization Vaccines and Biologicals (IVB Catalogue 2014). http:// www.who.int/immunization/documents/en (accessed 16 June 2014).

17. World Health Organization. Immunization profile - South Africa. 2011. http://apps.who.int/ immunization_monitoring/globalsummary/wucoveragecountrylist.html (accessed 16 June 2014).

18. Cui F, Liang X, Gong X, et al. Preventing hepatitis B though universal vaccination: Reduction of inequalities through the GAVI China project. Vaccine 2013;31(Suppl 9):J29-J35. [http://dx.doi. org/10.1016/j.vaccine.2012.07.048

19. Mphahlele MJ, Tshatsinde EA, Burnett RJ, Aspinall S. Protective efficacy and antibody follow-up of hepatitis B vaccine within the South African expanded programme on immunisation. S Afr Med J 2002;92(8):612-613.

20. Hino K, Katoh Y, Vardas E, Sim J, Okita K, Carman WF. The effect of introduction of universal childhood hepatitis B immunization in South Africa on the prevalence of serologically negative hepatitis B virus infection and the selection of immune escape variants. Vaccine 2001;19(28-29):39123918. [http://dx.doi.org/10.1016/S0264-410X(01)00121-9]

21. Tsebe K, Burnett RJ, Hlungwani NP, et al. The first five years of universal hepatitis B vaccination in South Africa: Evidence for elimination of HBsAg carriage in under 5-year-olds. Vaccine 2001;19(2829):3919-3026. [http://dx.doi.org/10.1016/S0264-410X (01)00120-7]

22. Schoub BD, Matai U, Singh B, et al. Universal immunization of infants with low doses of a low-cost plasma-derived hepatitis B vaccine in South Africa. Bull World Health Organ 2002;80(4):277-281.
.

23. Simani OE, Leroux-Roels G, François G, Burnett RJ, Meheus A, Mphahlele MJ. Reduced detection and levels of protective antibodies to hepatitis B vaccine in under 2-year-old HIV positive South African children at a paediatric outpatient clinic. Vaccine 2009;27(1):146-151. [http://dx.doi.org/10.1016/j. vaccine.2008.10.004]

24. Burnett RJ, Ngobeni JM, François G, et al. Increased exposure to hepatitis B virus infection in HIV-positive South African antenatal women. Int J STD AIDS 2007;18(3):152-156. [http://dx.doi. org $/ 10.1258 / 095646207780132523]$

25. Jones CE, Naidoo S, De Beer C, Esser M, Kampmann B, Hesseling AC. Maternal HIV infection and antibody responses against vaccine-preventable diseases in uninfected infants. JAMA 2011;305(6):576584. [http://dx.doi.org/10.1001/jama.2011.100]

26. Andersson MI, Maponga TG, Ijaz S, et al. The epidemiology of hepatitis B virus infection in HIV-infected and HIV-uninfected pregnant women in the Western Cape, South Africa. Vaccine 2013;31(47):5579-5584. [http://dx.doi.org/10.1016/.jvaccine.2013.08.028]

27. Wu TW, Lin HH, Wang LY. Chronic hepatitis B infection in adolescents who received primary infantile vaccination. Hepatology 2013;57(1):37-45. [http://dx.doi.org/10.1002/hep.25988]

28. Hipgrave DB1, Maynard JE, Biggs BA. Improving birth dose coverage of hepatitis B vaccine. Bull World Health Organ 2006;84(1):65-71. [http://dx.doi.org/10.2471/BLT.04.017426]

Accepted 25 July 2014 